\title{
Recirculating flows analysis and estimation inside channels
}

\author{
Christina G. Georgantopoulou ${ }^{1 *}$, Nikolaos S. Vasilikos ${ }^{1}$, George A. Georgantopoulos ${ }^{2}$ \\ ${ }^{1}$ School of Engineering, Bahrain Polytechnic, Isa Town, PO Box 33349, Kingdom of Bahrain \\ ${ }^{2}$ Aerodynamics and Flight Mechanics Sector, Hellenic Air Force Academy, Dekeleia Attika, Greece
}

\begin{abstract}
The recirculation which is developed during the flows inside pipes present a high interest in many industrial applications. In the present paper, a Cartesian grid method is presented which can be applied in pipes geometry approximation, even if the solid bounds are not lying on grid lines. A refinement technique using rectangular nested sub-girds is applied in order to avoid the unnecessary grid cells in the areas with no particular flow interest and cluster the grid when is needed. Important and useful for the industries results are extracted by these numerical simulations and estimations regarding the exact position and extend of the recirculation zones and the relating points. The estimation is taking placefor incompressible laminar, viscous flows inside inclined step channelsfor a range of inclination angles and Reynolds numbers values. The Navier - Stokes equations are solved using the artificial compressibility method according to the necessary boundary conditions arrangement. Flow results are presented for several grid sizes and Reynolds numbers focused on the recirculationzones length, in upper and lower channel' walls. Accepted accuracy of the flow results is produced using the aforementioned refinement algorithm, while the flow zones can be located according to the inlet flow rate, in order to avoid possible problems in the industries as corrosion or energy losses.
\end{abstract}

\section{Introduction}

The computational models are flexible tools for the industrial flow applications as useful results can be extracted for the optimization of the production line. The case of sudden expansion channels is also quite common in various industrial applications. For this reason, several research attempts can be found regarding the solution of the flows inside this type of channels [1,2] or the validation of their results using experimental methods $[3,4]$.

The grid generation approach is also important in these cases, as a huge number of cells can be createddue to the desired accuracy. Although several grids can be applied providing the necessary accuracy, the Cartesian grids seem to be more attractive due to the simple arrangement, easy specification of the geometry description and not complicated equations discretization $[5,6]$. In many channels' flow simulation and estimation cases, the uniform Cartesian grid requires high computational memory without any benefit to the accuracy of the results. In these cases, a refinement technique must be applied, according to the geometrical bound description and flow field requirements $[7,8,9]$.

In the present paper, the flow inside sudden expansion channels are simulated and estimated as well. The main results are not limited only to velocity or pressure distribution, but also to locate the exact position of the recirculation zone extend at the upper and lower wall of the channels. It is expected such a flow pattern due to the sudden step inside the channels. The grid methodology is developed using only Cartesiangrid lines, which is quite acceptable for such geometries providing robust results [10]. This method is based on Tseng approach[11], while it has been already applied successfully for pipe flows prediction in our recent research work [12]. A refinement technique is required to be developed, as grid clustering is required only to specific areas with flow interest. Rectangular nested grids are generated, where the numerical simulation is taking placeafter the necessaryboundary conditions applications along the common edges $[13,14]$. The flow Navier - Stokes (NS) equations are solved using the flux vector splitting methodology in combination with an artificial compressibility algorithm [15]. The final results

*Corresponding Author: Christina@polytechnic.bh 
provide acceptable accuracy and have been validated with other computational techniques and literature corresponded ones.

\section{Mathematical and Numerical approach}

\subsection{Channel geometry and flow equations}

The channels' geometry is easy to be projected due to the most bounds' alignment with the cartesian grid lines. The original channel geometry is projected onto the cartesian grid lines and a set of points is set accordingly. These points are lied either along the $\mathrm{x}$ either on y-axis. The rule of minimum distance is used in order to "transfer" these points on the grid nodes (Fig. 1). The new set of points (on the grid nodes) are the approximate geometry points and define a new sawtooth channel's bound. These points can be connected using straight lines by two ways: using Cartesian grid lines or diagonal lines. In our paper the Cartesian grid lines are chosen as the saw-tooth method is applied.

As we have initially mentioned, the geometry approximation is simple in the channels' cases, as all the bounds are aligned with Cartesian lines apart of one. Only for this part of the geometry, approximated points and bound will be created. The more clustering the grid, the more accurate approximated bound is extracted. This technique has successfully been applied in our previous work and this is the main reason that we choose to develop it for these cases as well $[12,16,17]$.

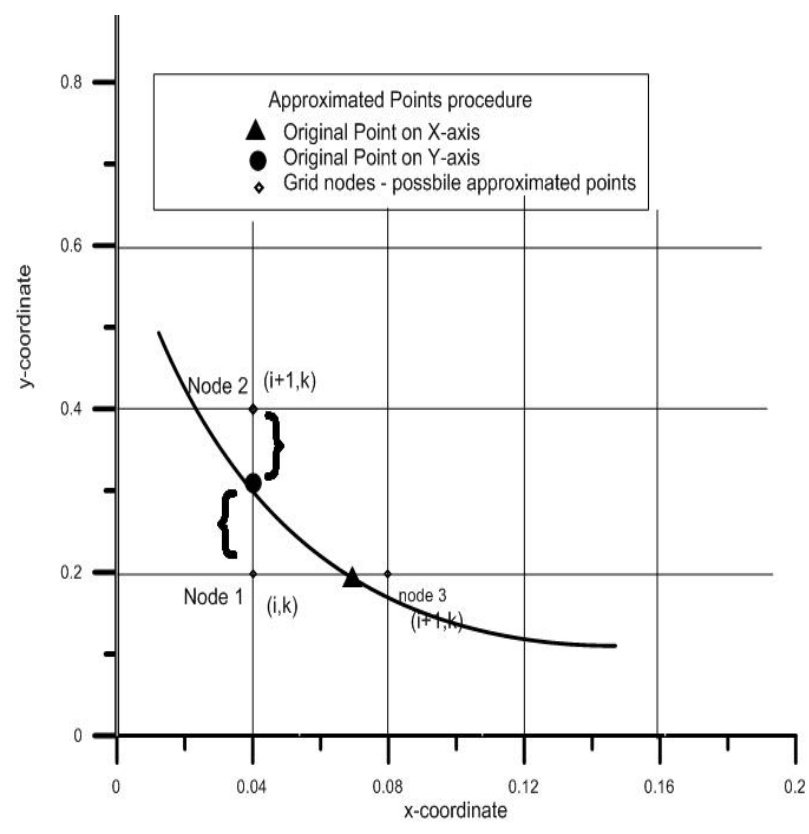

Fig. 1. Approximated points definition and procedure
Although, this flow numerical estimation case, seems not to require ununiform grids, the high number of computational cells increase the required computational memory without offering any benefit to the accuracy of the results. This effect is even worst in the case of unsteady or turbulent flows. There are several refinement techniques that can be applied but according to the simplicity of the channel geometry a rectangular nested technique is developed which produced accurate results despite of the fact that the flow variables values are transferred among the sub-grids. The method is based on using a hierarchical sequence of nested rectangular (block) meshes in which the numerical simulation is taking place (Fig. 2). In the beginning the flow solver procedure is applied to the initial grid, while the estimation is continued to the next level adjacent sub-grids. The integer refinement factor Iis defined, as follows:

$$
I=d x_{m} / d x_{m+1}=d z_{m} / d z_{m+1} .
$$

where $I$ is the refinement factor, $d x_{m}$ and $d x_{m+1}$ the grid's resolution in longitude for the $m$ grid level and for the $m+1$ (next) grid level, respectively, while $d z_{m}$ and $d z_{m+1}$ the grid's resolution in latitude for the grid levels as above. This method provides robust and accurate results minimizing the computational time as well $[16,17]$.

The flow inside the channels is received as laminar, steady, viscus and incompressible. For these reasons the flow is described by the Navier - Stokes equations as per below:

$$
\begin{gathered}
\frac{\partial \rho}{\partial t}+\frac{\partial(\rho u)}{\partial x}+\frac{\partial(r w)}{\partial z}=0(2) \\
{[\Gamma] \frac{\partial q}{\partial t}+\frac{\partial \mathrm{e}}{\partial \mathrm{x}}+\frac{\partial g}{\partial z}=\frac{1}{\operatorname{Re}}\left(\frac{\partial}{\partial \mathrm{x}}+\frac{\partial \mathrm{s}}{\partial z}\right)(3)} \\
{[\Gamma] \frac{\partial q}{\partial \mathrm{t}}+\frac{\partial \mathrm{e}}{\partial \mathrm{x}}+\frac{\partial g}{\partial z}+\alpha \cdot \frac{g_{1}}{z}=\frac{1}{\operatorname{Re}}\left(\frac{\partial r}{\partial \mathrm{x}}+\frac{\partial \mathrm{s}}{\partial z}+\alpha \cdot \frac{s_{1}}{z}\right)(4)}
\end{gathered}
$$

where,

$$
[\Gamma]=\operatorname{diag}\left(\frac{1}{\beta}, 1,1\right)^{\mathrm{T}}(5)
$$


and $\beta$ is the artificial compressibility term, $a$ is a switch for the activation of the axisymmetric terms $(\alpha=0$ is nonaxisymmetric, $a=1$ axisymmetric flow field), $R e$ the Reynolds number and $Q$ the unknown solution vector, as follows:

$$
Q=\left(\begin{array}{lll}
p & u & w
\end{array}\right)^{T}
$$

with $p$ being the pressure, and $(u, w)$ the velocity components in physical space, $e, g, g_{1}$ and $r, s, s_{1}$ are respectively the convective and diffusive flux vectors at the plane $(x, z)$.
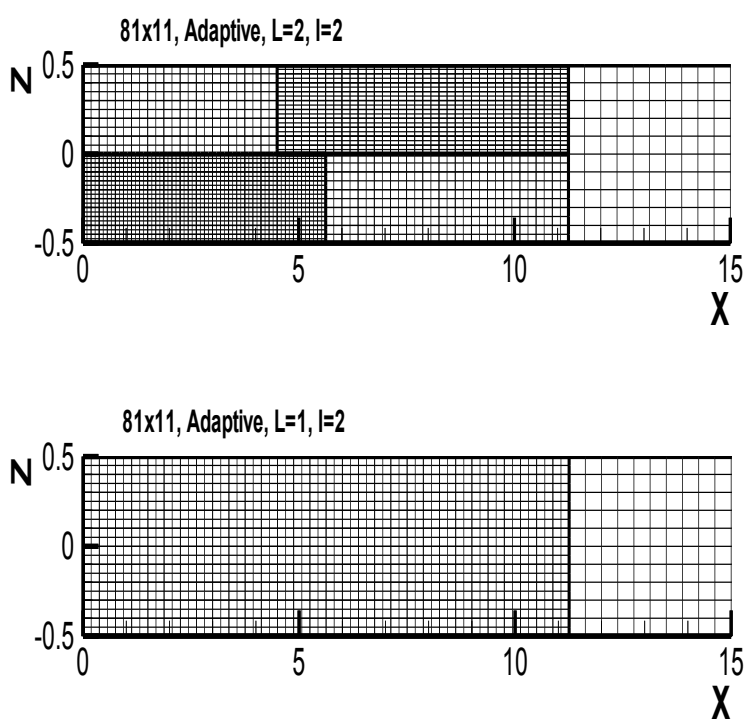

Fig. 2. Refinement technique for the channel geometry and grid generation. Grid size: $81 \times 11$, one (1) and two (2) levels of refinement, where $\mathrm{I}=2$.

As it can be easily seen, this type of governing equations is applied for unsteady flows.However, this form of the equations can be used for the solution of steady flow fields as in our case as we know that these are non-linear and iterative method are needed for their solution $[17,18]$.

\subsection{Boundary conditions}

In this flow case, two types of boundary conditions must be applied, either at the physical bounds, either at the neighboring grids bounds inside the channel. The relating conditions are presented at the below Table 1 with reference to Fig. 3, where $u$ and $w$ are the velocity components, $p$ the pressure and the indicator ref shows the corresponded reference values of the flow field.

Because of the sub-grids creation, the velocity and pressure values have to be transferred among the grids in order to ensure the consistent numerical estimation. If we assume that the velocity values have to be transferred from a coarse interface (grid level $=l_{i}$ ) to a refine one (grid level $l=l_{i+1}$ ), we receive:

$$
u_{l, m}=u_{l, m+1}=u_{i, j}
$$

where $u_{l, m}$ and $u_{l, m+1}$ are the axial velocity components at the refined grid level and $u_{i, j}$ the corresponded one at the coarse level.In the case of the physical bound conditions artificial (ghost) cells are considered, while these are neglected in the case of the sub-grids.

Table 1: Channel flow estimation boundary conditions

(Fig. 3)

\begin{tabular}{|c|c|}
\hline $\begin{array}{c}\text { Geometry } \\
\text { Bounds } \\
\text { (Fig. 4) }\end{array}$ & Boundary Conditions \\
\hline $\mathrm{AB}$ & Inlet conditions $u=0, \frac{\partial p}{\partial x}=0$ \\
\hline $\mathrm{AC}$ & No slip condition (wall) \\
$u=w=0, \frac{\partial p}{\partial z}=0$
\end{tabular}

At the specific flow case study in various sub-grids domains, the vertical velocity component is equal to zero (0) and so this is set at the aforementioned boundaries. However, even if $\neq 0$, the values will be transferred by a similar way with the axial velocity components as above: 


$$
w_{l, m}=w_{l, m+1}=w_{i, j}
$$

All the symbols are as these described above.

In the case that the boundary values must be transferred through fine - course interface the below formula is applied:

$u_{i, j}=\frac{u_{l, m+1}+u_{l, m}}{2}(9) \quad$ and $w_{i, j}=\frac{w_{l, m+1}+w_{l, m}}{2}(10)$

where the values are as described above.

In some cases, according for example the specific flow patterns inside the channels, fine grids are adjacent, and the variables values have to be transferred among them. The level of the grids receives the same value in both of the sub-grids and the velocity values are given as it can be seen below:

$$
u_{l, m}=u_{k, n} \quad \text { and } \quad w_{l, m}=w_{k, n}
$$

As the aforementioned grid method is applied only for rectangular Cartesian grids and sub-grids, there are some cases that course grids will be adjacent and the related boundary conditions must be set in order the numerical calculation to take place. In that case the boundaries application is developed as at the above case fine-fine interface (Eq. 11).

\section{Results}

The flow inside sudden expansion channels will be numerically analyzed, simulated and estimated. By presenting the following extended analysis we attempt to enhance previous research work which has been done on theseflow fields $[3,19]$ and extend our research work in incompressible viscous flows inside pipes [17, 18, 20] providing specific, accurate and useful results for the flow development.

The geometry description and the related dimensions can be seen at the above Fig.3. The relevant height $H_{\text {ref }}$ has set equal to 1 for dimensionless purposes and it is the maximum hydraulic diameter of the pipe. The inlet area receives $H_{\text {ref }} / 2$ height as we study a sudden expansion pipe.

In order to investigate the inclination angle effect to the flow, we present various calculations for three different inclination angles values: $30^{\circ}, 45^{\circ}$ and $90^{\circ}$ (Fig. 5), for five different $R e$ values: 100, 400, 800, 1200 and 1700 .

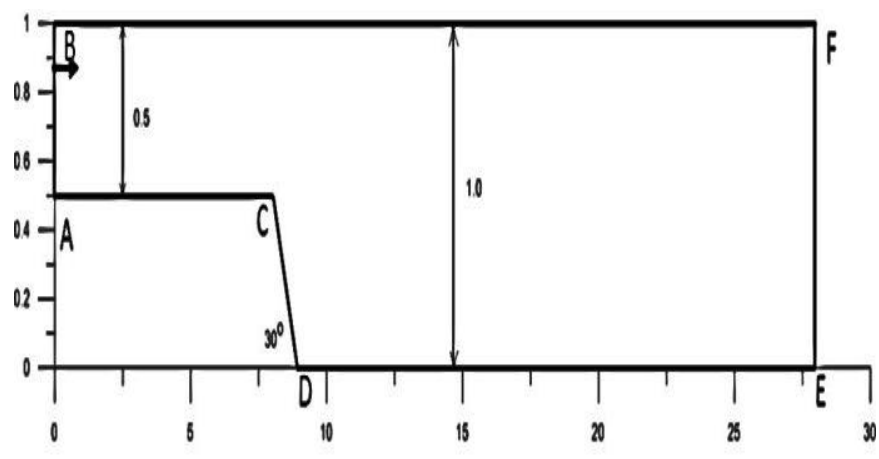

Fig. 3. Channel geometry description. The dimensionless lengths of the channel vary according to the $R e$ value.

\subsection{Recirculation lengths and points}

The results that are presented have been produced using grid size $351 \times 26$, with two refinement grid levels and $I=2$. In all the cases, the expansion ratio is set to be equal to 2 (Fig. 3). At the following tables the separation zones of the lower and the upper wall of the pipes are presented according to the inlet flow rate for the above $R e$ values. Detachment and reattachment points have been located in order to provide even more efficient information concerning the exact location of the separation zones.

For validation purposes, all the numerical cases solved using the commercial software ANSYS and some of these are presented for inclination angle equal to 90 $d e g$ for all the range of the used $R e$, using uniform grid sized $651 \times 26$.

In addition, the present work results are compared with the corresponded ones by the literature where the convergence is accepted.

Table 2: Recirculation lengths and relating points - lower

\begin{tabular}{|c|c|c|c|}
\hline $\operatorname{Re}$ & Upper Wall & & \\
\hline & $\begin{array}{l}\text { Recirculation } \\
\text { Length }\end{array}$ & $\begin{array}{c}\text { Detachment } \\
\text { Point }\end{array}$ & $\begin{array}{c}\text { Reattachment } \\
\text { Point }\end{array}$ \\
\hline 100 & No detection & No detection & No detection \\
\hline 400 & No detection & No detection & No detection \\
\hline 800 & 4.74 & 13.54 & 18.28 \\
\hline 1200 & 7.54 & 14.51 & 22.05 \\
\hline 1700 & 10.07 & 15.85 & 25.92 \\
\hline
\end{tabular}
wall: Inclination angle $30 \mathrm{deg}$. Grid, $351 \times 13, l=2, I=2$. 
Table 3: Recirculation lengths and relating points - lower wall: Inclination angle $30 \mathrm{deg}$, ANSYS - Fluent, Cartesian uniform grid $651 \times 26$.

\begin{tabular}{|c|c|c|c|}
\hline \multirow[t]{2}{*}{$\operatorname{Re}$} & \multicolumn{3}{|c|}{ Lower Wall } \\
\hline & $\begin{array}{l}\text { Recirculation } \\
\text { Length }\end{array}$ & $\begin{array}{l}\text { Detachment } \\
\text { Point }\end{array}$ & $\begin{array}{c}\text { Reattachment } \\
\text { Point }\end{array}$ \\
\hline 100 & 0.44 & 8.89 & 9.33 \\
\hline 400 & 4.28 & 8.28 & 12.56 \\
\hline 800 & 6.58 & 8.28 & 14.86 \\
\hline 1200 & 7.74 & 8.28 & 16.02 \\
\hline 1700 & 9.03 & 8.28 & 17.31 \\
\hline
\end{tabular}

Table 4: Recirculation lengths and related points - upper wall: Inclination angle $30 \mathrm{deg}$, ANSYS - Fluent, Cartesian uniform grid $651 \times 26$.

\begin{tabular}{|l|c|c|c|}
\hline $\boldsymbol{R} \boldsymbol{e}$ & \multicolumn{3}{|c|}{ Upper Wall } \\
\hline & $\begin{array}{c}\text { Recirculation } \\
\text { Length }\end{array}$ & $\begin{array}{c}\text { Detachment } \\
\text { Point }\end{array}$ & $\begin{array}{c}\text { Reattachment } \\
\text { Point }\end{array}$ \\
\hline 100 & No detection & No detection & No detection \\
\hline 400 & No detection & No detection & No detection \\
\hline 800 & 5.02 & 13.55 & 18.57 \\
\hline 1200 & 7.72 & 14.55 & 22.27 \\
\hline 1700 & 10.13 & 15.93 & 26.06 \\
\hline
\end{tabular}

Table 5: Recirculation lengths and related points - lower wall: Inclination angle $45 \mathrm{deg}$. Grid, $351 \times 13, \mathrm{l}=2, \mathrm{I}=2$.

\begin{tabular}{|c|c|c|c|}
\hline $\boldsymbol{R e}$ & & \multicolumn{2}{|c|}{ Lower Wall } \\
\hline & $\begin{array}{c}\text { Recirculation } \\
\text { Length }\end{array}$ & $\begin{array}{c}\text { Detachment } \\
\text { Point }\end{array}$ & $\begin{array}{c}\text { Reattachment } \\
\text { Point }\end{array}$ \\
\hline 100 & 1.24 & 8.10 & 9.34 \\
\hline 400 & 3.82 & 8.10 & 11.92 \\
\hline 800 & 6.68 & 8.10 & 14.78 \\
\hline 1200 & 8.99 & 8.10 & 17.09 \\
\hline 1700 & 10.86 & 8.10 & 18.96 \\
\hline
\end{tabular}

Table 6: Recirculation lengths and related points - upper wall: Inclination angle $45 \mathrm{deg}$. Grid, 351x13, l=2, I=2.

\begin{tabular}{|c|c|c|c|}
\hline $\boldsymbol{R} \boldsymbol{e}$ & \multicolumn{3}{|c|}{ Upper Wall } \\
\hline & $\begin{array}{c}\text { Recirculation } \\
\text { Length }\end{array}$ & $\begin{array}{c}\text { Detachment } \\
\text { Point }\end{array}$ & $\begin{array}{c}\text { Reattachment } \\
\text { Point }\end{array}$ \\
\hline 100 & No detection & No detection & No detection \\
\hline 400 & 1.78 & 11.49 & 13.27 \\
\hline 800 & 4.20 & 13.81 & 18.01 \\
\hline 1200 & 6.19 & 15.75 & 21.94 \\
\hline 1700 & 8.72 & 17.20 & 25.92 \\
\hline
\end{tabular}

As it is depicted by all the tables, there are mainly two separation zones the most, inside the channel $(R e<1700)$, while the recirculation at the upper wall cannot be detected when the Re is equal to 100 and 400 in most of the cases.As the flow rate increases, the recirculation length increases as well ason both channel's walls. However, although the detachment points at the lower wall remain at the same position for all the inlet velocities (around 8.26 except for the case of $R e=100$ ), the one at the upper wall is moving forward according to the related Re. Despite of this fact the recirculation length at the upper bound is increased as the $R e$ increases. For the value of $R e$ equal to 100 although the separation zones are not so extended, it seems that there is a low width recirculation at the upper surface of the pipe, which, when the Reis equal to 1700 , receives a significant value. In Tables $2-6$ the related information is presented for the inclination angle value equal to $30 \mathrm{deg}$.

Table 7: Recirculation lengths and related points - lower wall: Inclination angle $90 \mathrm{deg}$. ANSYS - Fluent, Cartesian uniform grid $651 \times 26$.

\begin{tabular}{|c|c|c|c|}
\hline Re & \multicolumn{3}{|c|}{ Lower Wall } \\
\hline Recirculation & $\begin{array}{c}\text { Detachment } \\
\text { Point }\end{array}$ & $\begin{array}{c}\text { Reattachment } \\
\text { Point }\end{array}$ \\
\hline 100 & 1.30 & 8.05 & 9.35 \\
\hline 400 & 3.35 & 8.05 & 11.40 \\
\hline 800 & 4.15 & 8.05 & 12.20 \\
\hline 1200 & 5.25 & 8.05 & 13.30 \\
\hline 1700 & 5.85 & 8.05 & 13.90 \\
\hline
\end{tabular}

Table 8: Recirculation lengths and related points - upper wall: Inclination angle $90 \mathrm{deg}$. ANSYS - Fluent, Cartesian uniform grid $651 \times 26$.

\begin{tabular}{|c|c|c|c|}
\hline $\boldsymbol{R} \boldsymbol{e}$ & \multicolumn{3}{|c|}{ Upper Wall } \\
\hline & $\begin{array}{c}\text { Recirculation } \\
\text { Length }\end{array}$ & $\begin{array}{c}\text { Detachment } \\
\text { Point }\end{array}$ & $\begin{array}{c}\text { Reattachment } \\
\text { Point }\end{array}$ \\
\hline 100 & No detection & No detection & No detection \\
\hline 400 & 1.20 & 11.55 & 12.75 \\
\hline 800 & 4.60 & 12.00 & 16.60 \\
\hline 1200 & 6.80 & 12.60 & 19.40 \\
\hline 1700 & 8.60 & 13.10 & 31.70 \\
\hline
\end{tabular}


The numerical estimation has been developed using both of the computational techniques (block - Cartesian and ANSYS) for validation purposes, where the convergence of the results is acceptable, presenting very low relative error $\left(4 \times 10^{-3}<\right.$ rel. error $\left.<5.5 \times 10^{-3}\right)$. It seems that the development of the recirculation in both of the pipe's walls is higher than the previous case (angle equal to $10 \mathrm{deg}$ ), the detachment point of the lower wall remains in constant position and the upper wall separation zone is moving forward according to the Re.

The present work numerical data have been retrieved by $351 \times 13$ grid size, $l=2$ and $I=2$. It seems that the convergence is acceptable since the relative errors receive very low values. However, in some cases, certain differences are depicted. These seem to be developed due to the different grid sizes, the different entrance pipe lengths or the differences to the outflow boundary conditions application.

Table 9: Recirculation lengths and related points lower wall: Inclination angle $90 \mathrm{deg}$, grid $351 \times 13, l=2, I=2$.

\begin{tabular}{|c|c|c|c|}
\hline $\boldsymbol{R} \boldsymbol{e}$ & \multicolumn{3}{|c|}{ Lower Wall } \\
\hline & $\begin{array}{c}\text { Recirculation } \\
\text { Length }\end{array}$ & $\begin{array}{c}\text { Detachment } \\
\text { Point }\end{array}$ & $\begin{array}{c}\text { Reattachment } \\
\text { Point }\end{array}$ \\
\hline 100 & 1.17 & 8.05 & 9.22 \\
\hline 400 & 3.71 & 8.05 & 11.76 \\
\hline 800 & 6.25 & 8.42 & 14.67 \\
\hline 1200 & 7.53 & 8.45 & 15.98 \\
\hline 1700 & 8.12 & 8.50 & 16.62 \\
\hline
\end{tabular}

Table 10: Recirculation lengths and related points upper wall: Inclination angle $90 \mathrm{deg}$, grid $351 \times 13, l=2, I=2$.

\begin{tabular}{|c|c|c|c|}
\hline $\boldsymbol{R} \boldsymbol{e}$ & \multicolumn{3}{|c|}{ Upper Wall } \\
\hline & $\begin{array}{c}\text { Recirculation } \\
\text { Length }\end{array}$ & $\begin{array}{c}\text { Detachment } \\
\text { Point }\end{array}$ & $\begin{array}{c}\text { Reattachment } \\
\text { Point }\end{array}$ \\
\hline 100 & No detection & No detection & No detection \\
\hline 400 & 1.72 & 11.55 & 13.27 \\
\hline 800 & 4.40 & 12.75 & 17.15 \\
\hline 1200 & 6.75 & 15.15 & 21.90 \\
\hline 1700 & 7.85 & 16.01 & 23.86 \\
\hline
\end{tabular}

As we can see by the following figures, no detection of recirculation is presented in very low $R e$, while the appearance of the upper bound recirculation depends on the $R e$ values and the inclination angle as well (Fig. 6). Through these results, the influence of the step angle to the flow pattern can be specified. It seems that the higher step angle, the longest recirculation zone at the lower

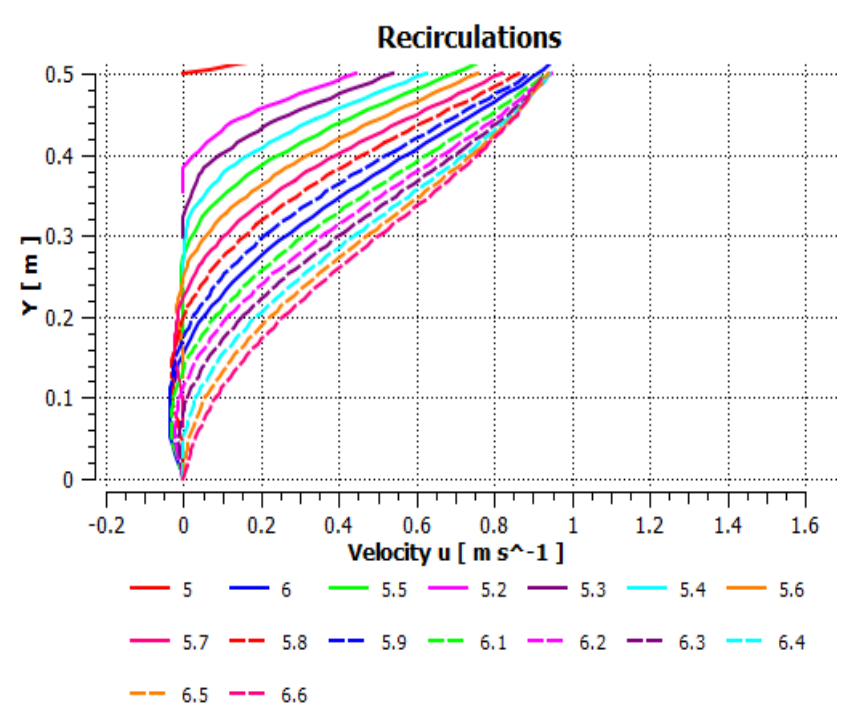

channel wall. By the other hand, the flow detachment on both channel walls is not the same (Fig. 7 and 8).

As we can identify by the Fig. 8, the more the inclination angle is increased the less extension of the upper wall recirculation is developed through the pipe if we assume that the Re remains constant. However, again at the highest Re values, the more extended lengths are

Fig 4. Lower wall recirculation inside the channel. $\mathrm{Re}=100$, inclination angle $=30^{\circ}$. ANSYS solution.

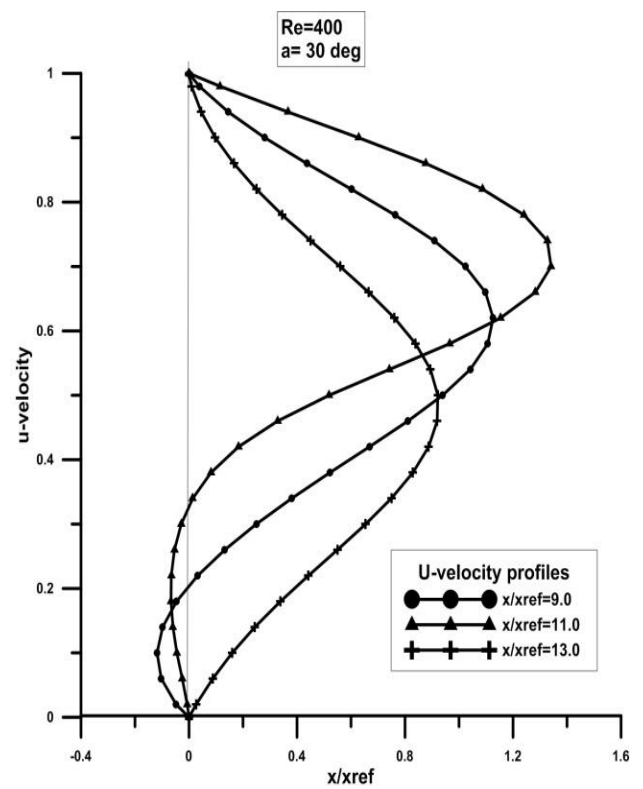

Fig. 5. Velocity profile along the pipe; $\mathrm{a}=30 \mathrm{deg}$ and $\mathrm{Re}=400$. 


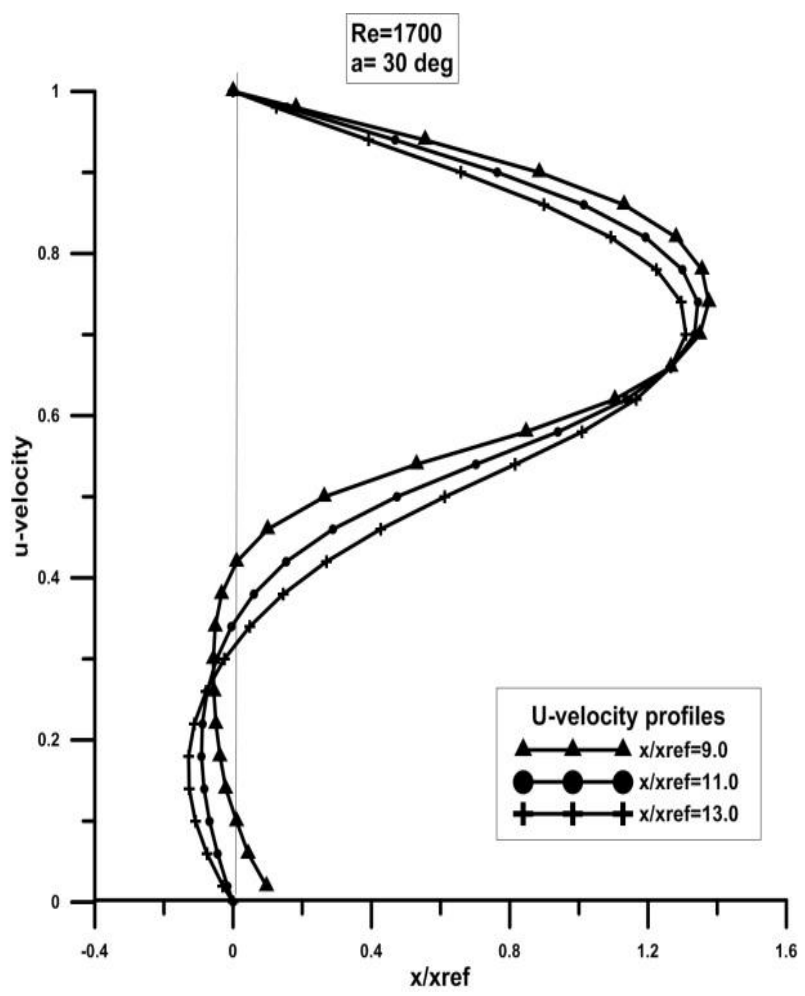

Fig. 6. Velocity profile along the pipe; angle $=30 \mathrm{deg}$ and $\mathrm{Re}=1700$.

presented. It is important that this relationship is followed by the flow in all the various Re and by this way we may conclude that this relationship applies for all the range of Reynolds number of laminar flow.

However, as we have concluded after several test cases in this field, the channel length downstream of the step affects the quality and the accuracy of the numerical results as sometimes the flow field is converged without oscillations. In our future research, the appropriate limits for this parameter requires further investigation, ensuring that every solution is length independent [21].

We agree with most of the researchers, who they claim that the entrance length of the pipe affects the numerical solution in low $\operatorname{Re}[22,23]$. Accordingtothe literature an entrance length more than 6 dimensionless ones should be enough in order not to affect the flow distribution inside the pipe. In order to create an independent solution we have applied eight (8) lengths for the entrance pipe and it seems that there is no influence along the fluid flow field.

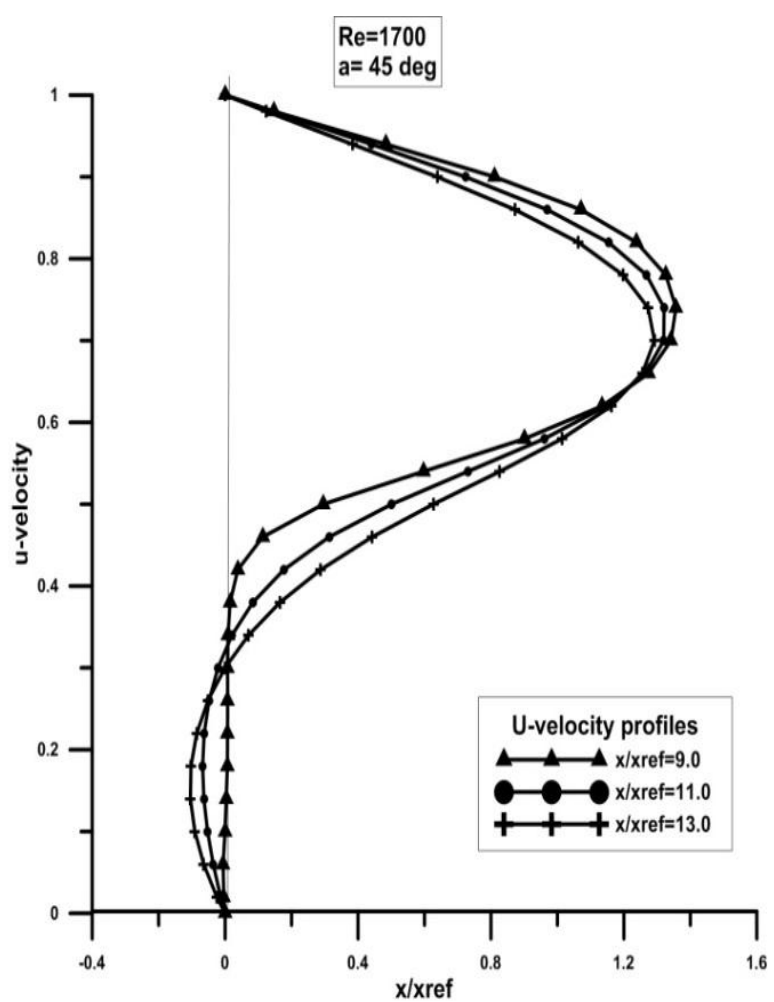

Fig 7. Velocity profiles; $\mathrm{a}=45 \mathrm{deg}$ and $\mathrm{Re}=1700$.

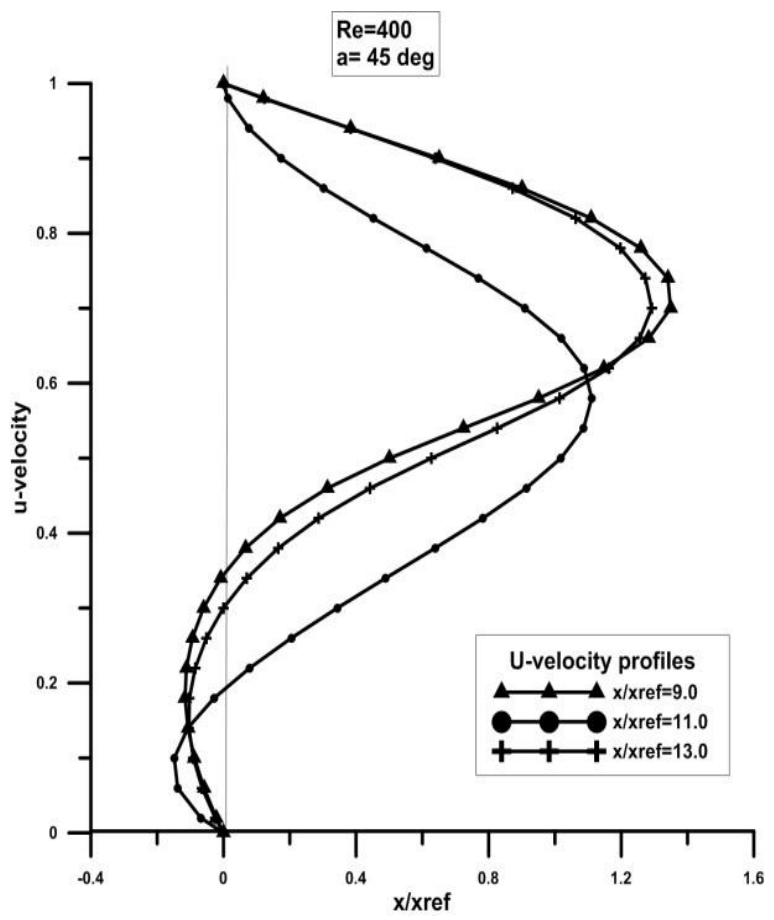

Fig. 8. Velocity profiles; $a=45 \mathrm{deg}$ and $\mathrm{Re}=400$. 


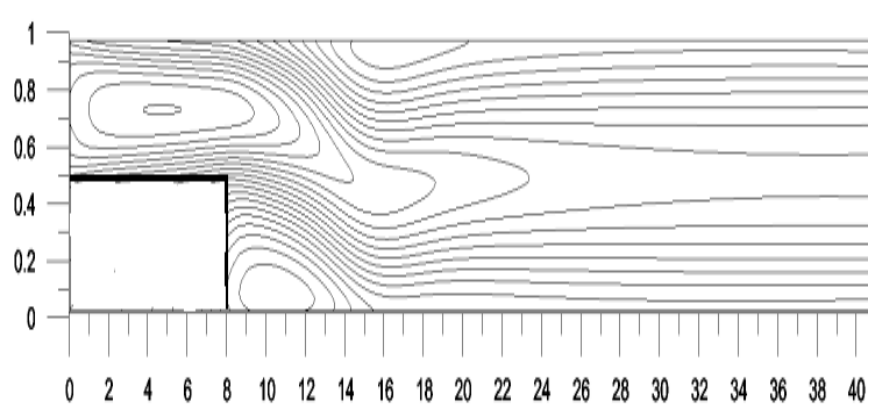

Fig. 9. Velocity contours along the pipe. Block nested grid, $\mathrm{I}=2, \mathrm{l}=2 . a=90^{\circ}$ and $R e=800$.

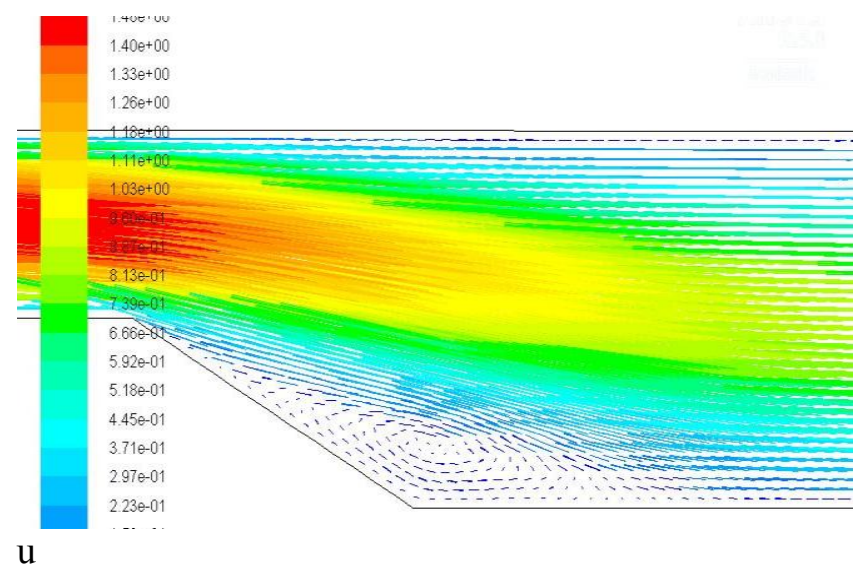

Fig. 11. Velocity contours along the pipe. ANSYS solution, $\mathrm{a}=30^{\circ}$ and $\mathrm{Re}=100$.

The overall flow development inside the channel can be seen through the velocity contours (Fig. 9-11) for various step angles and $\mathrm{Re}$ values. It seems that there is a good and acceptable convergence among the current work and ANSYS results.

\section{Conclusions}

The sudden expansion channel steady, laminar and viscus flows have been numerically analyzed, simulated, estimated and presented for a range of $\mathrm{Re}$ values and inclination angles. The flow characteristics, variables distribution, certain data information and points of upper and lower bound separation zones have been analytically presented for various values of $R e$. For the physical domain discretization, we use Cartesian grids, despite of the non-Cartesian bounds existence and in order to
In fig. 5 to 8 , the axial velocity profiles are presented for various a and Re values, while velocity profiles and recirculation graphs are presented below with the ANSYS solver as well for $\mathrm{Re}=100$ and $\mathrm{a}=30 \mathrm{o}$. As it was expected the profile is parabolic inside the pipe, while in the certain $\mathrm{x}$-coordinate location the separation areas are developed. It can be seen that the mass flow rate is conserved as well as the development of the flow is not affected by the downstream and upstream pipe's lengths.

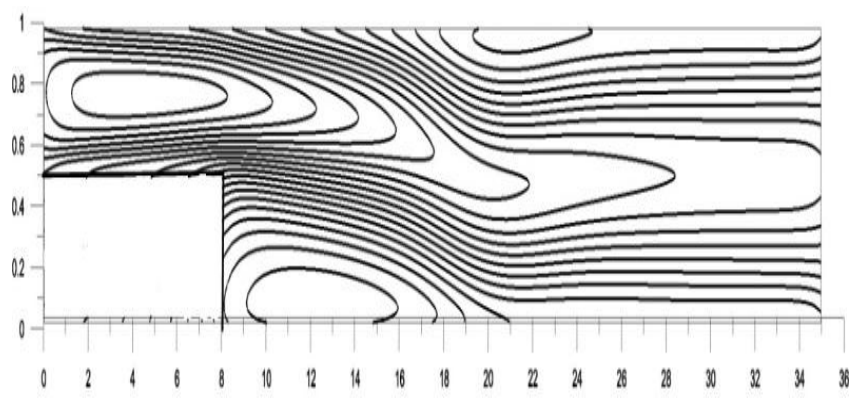

Fig. 10. Velocity contours along the pipe. Block nested grid, $\mathrm{I}=2, \mathrm{l}=2 \cdot a=90^{\circ}$ and $\mathrm{Re}=1200$.

overcome the high computational memory which is needed due to the grid cells number and reduce the computational time without decreasing the accuracy of the solution, we develop a block-nested refinement algorithm, which can be easily applied to the channel flow domain. It seems that if the $R e$ is higher than 400 and less than 1700, two separation zones are developed along the pipe in both bounds. The related detachment and reattachment points are clearly presented for every case and the final results show that in most of the cases the higher $R e$ the more extended recirculation area for a certain inclination angle. The most interesting and useful conclusion is the influence of the step angle of the pipe to the separation zones extend and development, which seems to be not the same for both pipe's bounds (upper and lower). More specifically, as the step angle is increased, it seems that the recirculation length at the lower wall is increased as well, for all therange of Re values. It's worth mentioning, that our results have been validated for various $R e$ values and grid sizes uniform and ununiformed as well, either by the corresponded ones by the literature either by the commercial ANSYS 
software ones. Further research is required regarding the experimental investigation of these flows taking into consideration the viscosity effect as well to the flow development and behavior inside the channel.

\section{References}

1 Santos R., Oliveira K., Figueiredo J., Mech.Comp, vol. XXIX, pp. 3347-3358, (2010).

2 Lee T. and Mateescu D. J. of fluids and structures, vol. 12, pp. 703-716, (1998).

3 Armaly B., Durst F., Pereira J., and Schounung B. J. of Fluid mech., vol. 127, pp. 473-496, (1983).

4 Coirier, W.J. and Powell, K.G., J. of Comp. Physics, Vol. 117, pp. 121-131, (1995)

5 Georgantopoulou C, Pappou Th., Tsangaris S.G., Proc. of the 4th GRACM conf. on Comp. Mech., pp. 526-534, (2002)

6 Wang, Z.J., Computers and Fluids, Vol. 27, pp.529549, (1998)

7 Karai E., Kultari A., Haluk M., Hydrology and Ecology, Vol. 1 pp. 15-25

8 Agresar, G., Linderman J.J., Tryggvason, G. and Powell, K.G., J. of Comp. Physics, Vol. 143, pp. 346380, (1998)

9 Georgantopoulou C., Georgantopoulos G., Vasilikos N., Int. J. of Mathematical Models and Method in Applied Sciences, 9 248-260, (2015).

10 Tseng Y., Ferziger J., J. of Computational Physics, vol.192, pp. 593-623, (2003)

11 Georgantopoulou C., Georgantopoulos G., Vasilikos N., WSEAS Transactions on Fluid mechanics, 10 6979, (2015) .

12 C. Georgantopoulou and S. Tsangaris, Applied Mathematical Modeling, Vol.31, pp2136-2148.

13 Barton Ie., Int. J.Num.Methods Fluids, vol. 25, 663644, (2015).

14 Berger M.J. and Collela P., J. of Comp. Physics, Vol. 83, pp.64-84, 1989.

15 Pappou, Th. and Tsangaris, S., Int. J. for Num. Methods in Fluid, Vol. 25, pp.523-545, (1997)

16 Georgantopoulou C., Georgantopoulos G., Vasilikos N. and Tsangaris S., Proc. of Int. Conf. on Applied Mathematics, simulation, modeling, pp.256-263, (2010).

17 Georgantopoulou C., WSEAS Transactions on Fluid Mechanics, vol. 17, (2017).

18 Mavromatides, L., Michel, P., El Mankibi, M., \&Santamuris, M., Building Simulation, 3, 279-294. (2010).

19 Biswas G., Breuer M., Durst, Transactions of ASME, vol. 126, pp. 362-374, (2004)

20 C. Georgantopoulou, Feras Ali, N. Vasilikos, G. Georgantopoulos, Applied Mechanics and Materials, vol. 829, pp. 15-20, (2016).

21 Kanfoudi H., Zgoli R., Int. J. of Mod., Simulation and Scientific Comp., vol. 6, (2015).

22 Erturk E.,Computers and Fluids, vol. 37, pp. 633655, (2008).

23 Gartling D, Int. J. Num. methods, vol. 11, pp. 953967, (1990). 